\title{
Effects of Weight Loss on Sweet Taste Preference and Palatability following Cognitive Behavioral Therapy for Women with Obesity
}

\author{
Tomoe Nishihara $^{a}$ Takehiro Nozaki ${ }^{a}$ Ryoko Sawamoto ${ }^{a}$ Gen Komaki ${ }^{b}$ \\ Noriyuki Miyata $^{a}$ Masako Hosoi ${ }^{a}$ Nobuyuki Sudo ${ }^{a}$ \\ ${ }^{a}$ Department of Psychosomatic Medicine, Graduate School of Medical Sciences, Kyushu \\ University, Fukuoka, Japan; ${ }^{\text {b}}$ Faculty of Medical Science, Fukuoka International University of \\ Health and Welfare, Fukuoka, Japan
}

\section{Keywords}

Obesity $\cdot$ Weight loss $\cdot$ Sweet taste $\cdot$ Cognitive behavioral intervention

\begin{abstract}
Objective: Current evidence suggests that obesity is associated with alteration of sweet taste perception. The purpose of this study was to determine if nonsurgical cognitive behavioral therapy (CBT)-based weight loss can cause a change in sweet taste perception. Methods: This case-control study consisted of 51 women aged 21-64 years. Twenty-seven with obesity or overweight were assigned to an obesity (OB) group (BMl: $29.8 \pm 0.5 \mathrm{~kg} / \mathrm{m}^{2}$ ) and 24 to a normal control (NC) group (BMl: $20.9 \pm 0.3 \mathrm{~kg} / \mathrm{m}^{2}$ ). The OB group underwent a 30 -week weight loss intervention using CBT-based group therapy. The results of measurement of detection threshold, suprathreshold perceived intensity, preference, and palatability, elements of sweet taste perception, were compared before and after the intervention. Psychological variables and appetite-related hormonal levels were measured. Results: Twenty-three patients and 22 controls completed the study. The OB group showed a $14.6 \%$ weight loss after the intervention. At baseline, the OB group preferred significantly higher sucrose concentrations than did the NC group; however, this difference was no longer significant after intervention. In the $O B$ group, persistent pleasure and reduced desire for other taste, measured by repeated exposure to sweetness, normalized after weight loss to levels comparable to those seen in the NC group. No significant difference in discriminative perception of the threshold concentration or the suprathreshold sensory value was found between the two groups before or after intervention. A significant correlation was found between the basal preferred sucrose concentration and the serum leptin level of the $O B$ group after adjusting for confounding factors, such as BMI, depressive symptom score, and trait-anxiety scores. Conclusions: Weight loss in-
\end{abstract}


Nishihara et al.: Effects of Weight Loss on Sweet Taste Preference and Palatability

duced by CBT-based nonsurgical intervention resulted in the normalization of the sucrose preference and palatability of women with obesity. Leptin activity may be associated with the altered sweet taste preference of people with obesity.

(C) 2019 The Author(s)

Published by S. Karger AG, Base

\section{Introduction}

Obesity is a multifactorial disease determined mainly by lifestyle factors, such as food intake and physical activity. As the prevalence of obesity and related diseases continues to grow, prevention through nutritional and lifestyle modification is imperative. In order to offer more specific and efficacious recommendations and interventions, a better understanding of the mechanisms underlying increased calorie intake and/or unbalanced diet is needed.

Current evidence suggests that sweetened foods have an impact on the development of obesity, with a recent review concluding that sugar-sweetened beverage consumption is positively associated with obesity indices, both in children and adults [1]. Eating behavior is motivated both by the need to restore homeostasis and by a preference for food with high fat and/ or sugar content. Moreover, a recent study demonstrated that sweet hedonic liking correlated positively with total energy and carbohydrate intake measured by food records [2].

Many studies have investigated if individuals with obesity have different sweet taste perception than normal-weight individuals. Some have reported that obesity is associated with alteration in sweet taste sensitivity, which could contribute to increased food intake [3]. Bartoshuk and colleagues used the general Labeled Magnitude Scale (gLMS) procedure [4] to show that individuals with obesity experience decreased sweetness of food and an increased liking for sweetness compared to individuals of normal weight [5]. Furthermore, recent studies have reported that the hedonic component of sweet taste perception decreased after Roux-en-Y gastric bypass (RYGB) and that sleeve gastrectomy induced weight loss [6, 7]. Several mechanisms, such as hormonal or anatomical changes resulting from the operative procedure, have been proposed to explain the decrease in perceived sweetness. However, it is unclear if the change in taste perception is a surgery-specific phenomenon or if it is a general phenomenon that accompanies weight loss.

The primary goal of this study was to determine if nonsurgical weight loss would also change sweet taste preference and palatability. We tested a group of women with obesity $(\mathrm{OB})$ and a normal-weight control (NC) group for sweet taste perception before and after cognitive behavioral therapy (CBT)-based weight loss by the OB group.

\section{Materials and Methods}

\section{Participants}

The participants were recruited through an advertisement posted in the local newspaper. The investigators provided the details of the study and answered questions from individuals who were interested in participating and who met the eligibility requirements. The exclusion criteria were as follows (1): diagnosed with a taste disorder (2); history of sinusitis or any current sinus-related problems (3); weight loss of over $5 \mathrm{~kg}$ in the previous 6 months (4); history of bulimia nervosa or anorexia nervosa (5); pregnant or breast-feeding during the study period or intention to become pregnant within 2 years of the study (6); use of any medication or supplement that might affect weight or taste (7); history or current diagnosis of an illness that might affect weight, e.g. malignancy or endocrine disease (8); use of medication for the 
Fig. 1. Study flow diagram. The obesity (OB) and control (NC) groups underwent evaluation including the measurement of taste perception before and after weight loss by the OB group. Four participants from the OB group dropped out due to the following reasons: Unwillingness to follow the program $(n=2)$, family problems $(n=1)$, and pregnancy during the 7-month weight loss period $(n=1)$. Two participants from the NC group were unable to participate in the second evaluation, while the remaining 22 individuals completed the second evaluation.

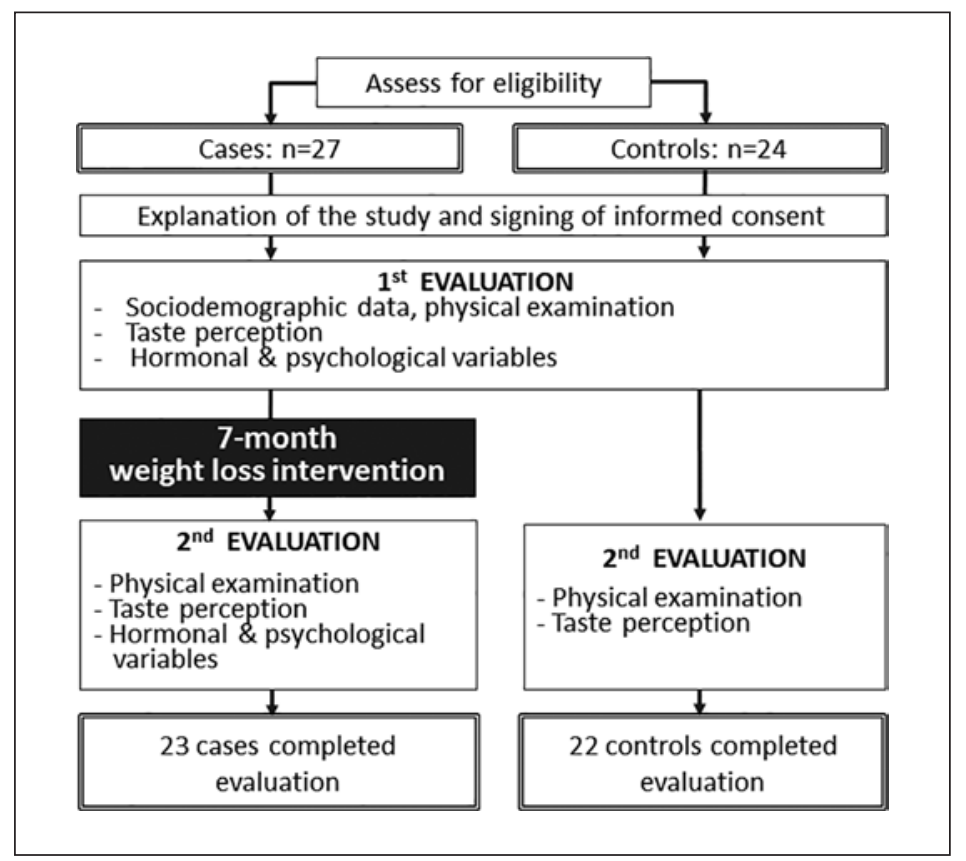

treatment of psychiatric disease (9); intention to move or relocate during the research period. Of the 51 women, aged 21-65 years, who met the criteria, 27 with a body mass index (BMI) between 25 and $40 \mathrm{~kg} / \mathrm{m}^{2}$ were placed in an obesity group (OB) and 24 with a BMI between 19 and $24 \mathrm{~kg} / \mathrm{m}^{2}$ were placed in a normal control group (NC). Weight and height measurement was done with a fully automatic measuring system (Francics MX-300). The enrolment period lasted from September 2015 to June 2017. Figure 1 illustrates the flow of the study. Demographic, lifestyle, and health history data were collected from each of the participants.

\section{Experimental Procedures}

The tests were performed after fasting for $12 \mathrm{~h}$ overnight at home. The OB participants visited the Department of Psychosomatic Medicine of Kyushu University Hospital for assessment before and after the 7-month weight loss program. The NC group was tested in the same schedule. Data related to menstrual status and smoking habits were also obtained because of their potential to affect taste perception $[8,9]$.

\section{Taste Stimuli}

For threshold testing, sucrose solution was prepared in quarter-log dilution steps, with concentrations ranging from 1 to $1 \times 10-{ }^{4} \mathrm{M}$. To assess the suprathreshold intensity of taste perception, we used $0,0.09,0.36$, and $1.05 \mathrm{M}$ sucrose solutions. For preference testing, 0.09, $0.18,0.35,0.7$, and $1.08 \mathrm{~m}$ sucrose solutions were used. All solutions were prepared using deionized water and presented at room temperature $\left(20-22^{\circ} \mathrm{C}\right)$.

\section{Sensory-Discriminative Component of Sweet Taste Perception}

Detection Thresholds

The sucrose threshold concentration was determined through a two-alternative, forcedchoice staircase procedure $[10,11]$. Participants were presented with pairs of solutions, one of which was sucrose solution and the other deionized water. The concentration for the sucrose solution began at $1 \times 10-{ }^{4} \mathrm{M}$. After tasting each of the solutions without swallowing, the participants were asked to choose the one they thought contained the sweet taste. If their 
choices were incorrect, the level of the presented dilution step was increased. It was decreased after two consecutive correct responses. The procedure was terminated when four reversals occurred and the following two criteria were met [10]: (1) two successive reversals had occurred within two dilution steps; (2) the series of reversals could not form ascending patterns (one in which positive and negative reversals need to be achieved at successively higher concentrations). The threshold concentration was provided by the mean of the log values of the last four reversals.

\section{Suprathreshold Sensory Function}

The gLMS [4] was used to scale the perceived intensity of the suprathreshold concentrations of the series of concentrations of sucrose. After the participants were instructed about the use of the gLMS; two blocks of four concentrations for each taste were presented to the participant in a random order. After tasting each solution, the participant rated perceived intensity of sweetness on the gLMS scale. The estimate of the subject' taste intensity for each stimulus was provided by computing the mean value of intensity of each concentration during the two-block series.

\section{Hedonic Component of Sweet Taste Perception}

Preference Test

To determine the concentration of sucrose preferred by the participant, a forced-choice, paired-comparison tracking technique was used according to the previously reported methodology $[6,11,12]$. Participants were presented with pairs of solutions that differed in the concentration of sucrose and asked to taste both solutions for $10 \mathrm{~s}$, without swallowing. Participants rinsed their mouth with deionized water after tasting each solution. After tasting both of the solutions, they were asked to choose the one they preferred. They were first presented with a pair of samples with moderate concentrations (such as 0.18 vs. $0.35 \mathrm{M}$ ). After they choose one of them, subsequent presentations involved pairs of solutions consisting of (1) the solution chosen in the prior task; (2) a solution that was the successive higher or lower concentration. The procedure was continued until the participant had either chosen a solution of a specific concentration when it was paired with both higher and lower concentrations or had chosen the solution with the highest or lowest concentration. We then repeated the above task by presenting the pairs of solutions in the reverse order. Sucrose preference was estimated by computing the geometric mean of the concentrations of the final solution chosen in each trial.

Sweet Taste Palatability Test

The palatability of the sweet taste was measured using a validated experimental procedure as previously described $[6,13]$. Participants were asked to record the intensity of pleasantness of the taste and the desire for a different taste after tasting a $10 \mathrm{~mL}$ sample of a sucrose solution $(24 \% \mathrm{w} / \mathrm{v})$ for $10 \mathrm{~s}$, without swallowing. They rated the former using the hedonic version of the gLMS (ranging from -100 to $100 ;-100=$ strongest imaginable displeasure of any kind, $0=$ neural and $100=$ strongest imaginable pleasure of any kind) [14] and the latter using the regular gLMS (ranging from 0 to 100; $0=$ barely detectable and $100=$ strongest imaginable sensation of any kind). The trial was repeated 10 times at 2-min intervals, without any interstimulus rinse.

\section{Measurement of Appetite Hormonal Markers}

Fasting blood samples were drawn from a vein in the antecubital fossa between 10:00 a.m. and 12:00 noon. The separated serum samples were stored at $-80^{\circ} \mathrm{C}$ until they were analyzed. The serum hormone level was measured using commercially available kits. The serum leptin level was analyzed by radioimmunoassay (Hitachi Aloka Medical Ltd., Tokyo, 
Japan). The active ghrelin level was measured by enzyme-linked immunosorbent assay (Molecular Devices, Tokyo, Japan).

\section{Psychological Measurements}

Depressive symptoms and trait-anxiety scores have been reported to be inversely correlated with the sweet taste threshold [15]. Therefore, depressive symptoms were evaluated using the Japanese version of the Center for Epidemiologic Studies-Depression Scale (CES-D), for which the test-retest reliability and concurrent validity have been thoroughly documented [16]. The CES-D is a 20-item, self-report questionnaire, with scores ranging from 0 to 60 and higher scores indicating more severe depressive symptoms [17]. The anxiety levels were evaluated using the Japanese version of the State-Trait Anxiety Inventory (STAI) [18]. The STAI is a self-report questionnaire consisting of two scales: STAI-1 assesses state-anxiety levels, while STAI-2 assesses trait-anxiety levels. Each scale consists of 20 items that together indicate the presence or absence of anxiety symptoms [19].

The severity of binge eating was assessed using the Binge Eating Scale (BES) [20]. It comprises 16 self-administered items designed to measure the behavioral and emotional/cognitive symptoms associated with binge eating. Each item presents three or four differently weighted statements, with a final score varying from 0 to 46 . Eating behavior was also evaluated using the Japanese version of the Three Factors Eating Questionnaire (TFEQ), for which high consistency and construct validity have been confirmed for patients who are obese or overweight [21]. The 51-item TFEQ consists of three eating behavior factors; Restraint, Disinhibition, and Hunger [22]. We also assessed eating behavior using the Japanese version of the Dutch Eating Behavior Questionnaire (DEBQ), the validity and reliability of which have been evaluated in a previous study [23]. The DEBQ is a 33-item self-rated questionnaire that is divided into three subscales: restrained eating, emotional eating, and external eating [24]. Respondents rate each item on a 5 -point Likert scale, with scores ranging from 1 (seldom) to 5 (very often). Item scores for each subscale were added to obtain an overall subscale score, which was then divided by the number of subscale items to produce a final score between 1 and 5 .

\section{Weight Loss Intervention}

We used the CBT-based weight loss program for obesity originally developed by Cooper [25] and modified for use in our group therapy program, which was conducted in small groups consisting of 10-12 participants [26]. The program consisted of 26 group sessions and 4 individual sessions ( 26 for the weight reduction phase and 4 for the weight maintenance phase), conducted once a week for 7 months. The weight management sessions were conducted by two study physicians who were highly qualified psychotherapists with specialized training in CBT and by two certified dietitians. The sessions included the following three components:

\section{Diet Management}

In the first session, the participants were instructed to record all the foods and beverages they consumed, to measure the precise amounts on kitchen scales at home, and to write the results into a record sheet. They were also trained to calculate the energy of the specific food eaten using a food exchange list [27]. For the first 7-day diet record, the mean total energy and nutrient intakes were self-calculated and then confirmed through a recall interview by nutritionists. The regimen was composed of specific, prescribed nutritional policies to comply with guidelines [28]. The participants were instructed to consume a regular daily food diet containing $500 \mathrm{kcal}$ less than their baseline total calorie intake (approximately 1,400-1,500 kcal). The carbohydrate, protein, and fat balance was as follows: $50-60 \%$ of the daily calories were provided from carbohydrate, $15-20 \%$ from protein, and $20-30 \%$ from fat. The participants were expected to reduce sweets consumption to within $10 \%$ of the total intake. Also, it was recommended that 
Nishihara et al.: Effects of Weight Loss on Sweet Taste Preference and Palatability

they consume a minimum of 350 g per day of vegetables, divided equally among three meals, as described in the guidelines of the Ministry of Health, Labour and Welfare [29].

Physical Activity

Participants were encouraged to increase their level of physical activity. They did their own specific goal-setting at every weekly session after the 8th week and were encouraged to increase their walking steps gradually to moderate intensity levels (such as 8,000-10,000 steps/day).

Stress Management

Cognitive reconstruction and problem solving are the main components of our stress management program [30]. During the sessions, participants worked on issues concerning eating behaviors that might trigger excess calorie intake. This was done to relieve anxiety, compulsiveness, and emotional and impulsive eating.

\section{Statistical Analyses}

All continuous variables are expressed as mean \pm standard error, except for the preference concentrations, for which the median \pm interquartile range is used. All analyses were performed using the SPSS version $22.0 \mathrm{~J}$ statistical software package (IBM SPSS Statistics, Chicago, IL, USA).

Comparisons of changes in taste detection threshold, appetite hormonal markers, and nutrition-related or psychological variables were evaluated using the paired $t$ test after the data of participants who dropped out were removed. Nominal clinical data were analyzed by the $\chi^{2}$ test. Suprathreshold perception intensity data were positively skewed and required transformation into their square root values to approximate normal distribution. Two-way analysis of variance (ANOVA) was used to determine the difference between groups in the perceived intensity of suprathreshold concentrations. Sweet taste preference data were also skewed; thus, the Mann-Whitney U test was applied to compare the preference data between groups. To investigate correlation between sucrose preference and serum leptin level, sweet taste preference data were subjected to logarithmic transformation in order to indicate the normal distribution; then, Pearson correlation analysis and partial correlation analysis were applied to adjust for cofounding factors. The sweet taste palatability values at each trial were normalized with respect to the value at the first trial. Because they showed non-normal distribution, the Mann-Whitney U test was used to compare the difference in the first trial values of the groups. The normalized data were then compared with repeated measures ANOVA after exclusion of the extreme outliers based on the Smirnov-Grubbs' outlier test (measurements of two cases of the NC group were removed for the analysis of "pleasure elicited by sweetness," as were those of two cases in the OB group and two cases in the NC group for "desire for other taste"). To assess interaction effects, Greenhouse-Geisse correction was used when the Mauchly's test indicated that the assumption of sphericity had been violated. Bonferroni correction was applied to adjust for the simple main effect. Results with $p$ values less than 0.05 were considered significant.

\section{Results}

\section{Clinical Characteristics}

Baseline characteristics are shown in Table 1. The groups did not significantly differ in age, marital status, job condition, smoking habits, fasting serum level of glucose, or zinc level. The average body weight of the OB group decreased by $14.6 \%$ after the weight loss intervention. 
Table 1. Clinical characteristics

\begin{tabular}{|c|c|c|c|}
\hline & $\begin{array}{l}\text { Normal-weight women } \\
(n=24)\end{array}$ & $\begin{array}{l}\text { Women with overweight } \\
\text { or obesity } \\
(n=27)\end{array}$ & $p$ value \\
\hline Age, years & $45 \pm 1.6$ & $51 \pm 2.1$ & 0.052 \\
\hline \multicolumn{4}{|l|}{ Body weight, kg } \\
\hline Before weight loss & $51.9 \pm 1.1$ & $73.6 \pm 1.7$ & $<0.001^{*}$ \\
\hline After weight loss & $51.6 \pm 1.0$ & $61.7 \pm 1.3$ & $<0.001^{*}$ \\
\hline \multicolumn{4}{|l|}{ BMI, $\mathrm{kg} / \mathrm{m}^{2}$} \\
\hline Before weight loss & $20.9 \pm 0.3$ & $29.8 \pm 0.5$ & $<0.001^{*}$ \\
\hline After weight loss & $20.8 \pm 0.3$ & $25.1 \pm 0.4$ & $<0.001^{*}$ \\
\hline$\Delta$ Body weight, kg & -0.2 & -10.7 & $<0.001^{*}$ \\
\hline Percent weight loss & 0.4 & 14.6 & $<0.001^{*}$ \\
\hline Marital status - married, \% & 87.5 & 96.3 & 0.24 \\
\hline Job condition - worker, \% & 41.7 & 48.2 & 0.64 \\
\hline Smoker, \% & 4.2 & 3.7 & 0.93 \\
\hline \multicolumn{4}{|l|}{ Blood chemistry } \\
\hline Fasting blood glucose, mg/dL & $97.3 \pm 1.6$ & $99.7 \pm 3.2$ & 0.51 \\
\hline Serum zinc level, $\mu \mathrm{g} / \mathrm{dL}$ & $88.8 \pm 6.2$ & $82.4 \pm 1.8$ & 0.31 \\
\hline Serum leptin level, ng/mL & $11.6 \pm 1.2$ & $33.9 \pm 2.6$ & $<0.001^{*}$ \\
\hline Serum ghrelin level, fmol/mL & $24.5 \pm 3.4$ & $22.9 \pm 2.7$ & 0.70 \\
\hline
\end{tabular}

Values are presented as mean \pm SEM or as stated. $* p<0.05$, significant in intergroup comparison before treatment.

Psychological Variables, Energy Intake, and Nutrient Consumption

The BES disinhibition and hunger scores of the TFEQ and the emotional and external eating scores of the DEBQ were significantly higher in the OB group than in the NC group at baseline (Table 2). All except for the emotional eating score were significantly decreased after weight loss in the OB group, while the restrained eating scores of TFEQ and DEBQ were significantly increased (Table 2).

In the OB group, the average daily energy intake values were significantly lower after the weight loss intervention $(1,765 \pm 54 \mathrm{kcal}$ during the first week of intervention and 1,256 \pm 25 kcal during the last month of intervention, $p<0.001$ ), as were the average daily energy intake values from sweets ( $217 \pm 24$ and $78 \pm 5 \mathrm{kcal}$, respectively, $p<0.001)$.

\section{Sensory-Discriminative Component of Sweet Taste Perception}

We first examined the sucrose detection threshold. No significant difference was found between the groups before the intervention (NC: $9.9 \pm 1.2 \mathrm{~mm}$; OB: $11.0 \pm 1.4 \mathrm{~mm}, p=0.57$ ). No significant weight loss intervention-related change was detected in the sweet taste threshold of the OB group ( $p=0.99$ ).

As shown in Figure 2, the suprathreshold perception of taste intensity of both groups increased progressively with an increase in stimulus concentration, both before and after weight loss. The ANOVA revealed no significant interaction $(F(3,196)=0.14, p=0.94)$ and main effect $(F(1,196)=0.19, p=0.67)$ before intervention. Also, no significant interaction $(F(3,172)=0.44, p=0.72)$ or main effect $(F(1,172)=1.74, p=0.19)$ was found after weight loss. 
Fig. 2. Comparison of the suprathreshold perceived intensity of sweetness of the control (NC) and obesity (OB) groups. The NC (open circle) and OB (closed circle) groups underwent general Labeled Magnitude Scale (gLMS) evaluation before (A) and after (B) weight loss intervention for the OB group. Sqrt, square root. Data expressed as mean \pm standard error.

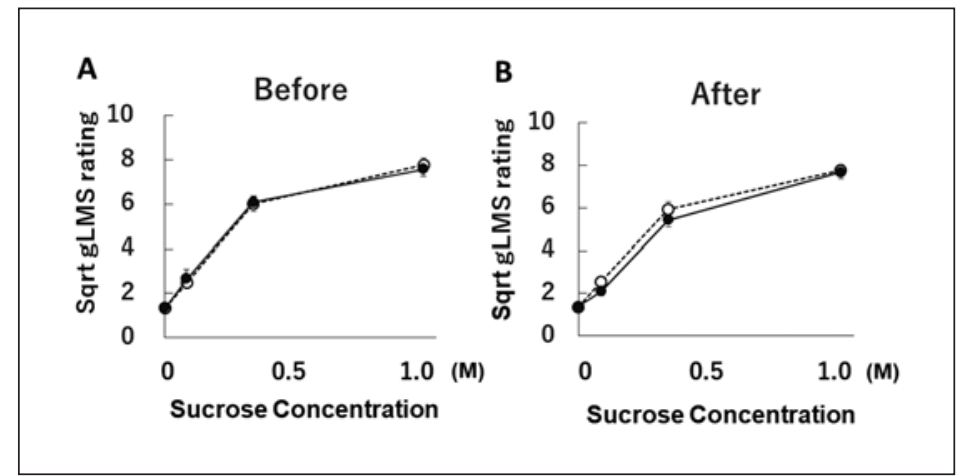

Table 2. Psychological variables (comparison between groups at baseline and before-after weight loss in the OB group)

\begin{tabular}{|c|c|c|c|c|c|c|}
\hline & \multirow{2}{*}{$\begin{array}{l}\text { Normal- } \\
\text { weight } \\
\text { women } \\
(n=24)\end{array}$} & \multirow{2}{*}{$\begin{array}{l}\text { Women with } \\
\text { overweight/ } \\
\text { obesity } \\
(n=27)\end{array}$} & \multirow[t]{2}{*}{$\begin{array}{l}p \text { value } \\
\text { intragroup }\end{array}$} & \multicolumn{2}{|c|}{$\begin{array}{l}\text { Women with } \\
\text { overweight/obesity }\end{array}$} & \multirow{2}{*}{$\begin{array}{l}p \text { vale } \\
\text { before- } \\
\text { after }\end{array}$} \\
\hline & & & & $\begin{array}{l}\text { before } \\
(n=23)\end{array}$ & $\begin{array}{l}\text { after } \\
(n=23)\end{array}$ & \\
\hline CES-D & $8.0 \pm 1.4$ & $11.5 \pm 1.6$ & 0.12 & $11.0 \pm 1.8$ & $9.7 \pm 1.5$ & 0.35 \\
\hline STAI-trait & $39.7 \pm 2.3$ & $42.8 \pm 2.1$ & 0.31 & $42.1 \pm 2.1$ & $38.4 \pm 2.2$ & 0.13 \\
\hline BES & $4.8 \pm 0.9$ & $12.7 \pm 0.9$ & $<0.001^{*}$ & $12.3 \pm 1.1$ & $7.6 \pm 1.1$ & $<0.001^{*}$ \\
\hline \multicolumn{7}{|l|}{ TFEQ } \\
\hline Restraint of eating & $8.7 \pm 0.8$ & $7.7 \pm 0.8$ & 0.35 & $8.1 \pm 0.9$ & $14.2 \pm 0.5$ & $<0.001^{*}$ \\
\hline Disinhibition & $4.6 \pm 0.5$ & $9.0 \pm 0.6$ & $<0.001^{*}$ & $8.9 \pm 0.6$ & $7.0 \pm 0.6$ & $<0.001^{*}$ \\
\hline Hunger & $3.8 \pm 0.6$ & $5.9 \pm 0.5$ & $0.01^{*}$ & $5.5 \pm 0.6$ & $3.5 \pm 0.5$ & $<0.001^{*}$ \\
\hline \multicolumn{7}{|l|}{ DEBQ } \\
\hline Restrained eating & $2.7 \pm 0.2$ & $2.8 \pm 0.1$ & 0.71 & $2.9 \pm 0.1$ & $3.8 \pm 0.1$ & $<0.001^{*}$ \\
\hline Emotional eating & $1.8 \pm 0.1$ & $2.3 \pm 0.2$ & $0.02^{*}$ & $2.2 \pm 0.2$ & $2.0 \pm 0.1$ & 0.17 \\
\hline External eating & $3.0 \pm 0.1$ & $3.3 \pm 0.1$ & $0.02^{*}$ & $3.3 \pm 0.1$ & $2.9 \pm 1.1$ & $0.001^{*}$ \\
\hline
\end{tabular}

Values are mean \pm SEM. $* p<0.05$, significant. CESD-D, Center for Epidemiologic Studies-Depression Scale; STAI, State-Trait Anxiety Inventory; BES, Binge Eating Scale; TFEQ, Three Factors Eating Questionnaire; DEBQ, Dutch Eating Behavior Questionnaire.

\section{Hedonic Component of Sweet Taste Perception}

Sweet Taste Preference

As shown in Figure 3, the OB group reported a preference for a higher sucrose concentration before weight loss than did the NC group ( $p=0.033$ ). However, no significant difference between the groups was found after weight loss intervention for the OB group $(p=0.43)$.

Sweet Taste Palatability

Figure 4 shows the scores for the pleasure elicited from tasting sweetness and the desire for other taste normalized to their values at the first trial. The "pleasure elicited by sweetness" values at the first trial were $11.3 \pm 5.0$ for $\mathrm{NC}$ and $-4.8 \pm 6.4$ for $\mathrm{OB}$ at baseline $(p=0.18)$. After weight loss, they were $4.4 \pm 8.1$ for $\mathrm{NC}$ and $2.5 \pm 7.6$ for $\mathrm{OB}(p=0.79)$. The "desire for other taste" values at the first trial were $4.8 \pm 1.4$ for $\mathrm{NC}$ and $17.2 \pm 3.7$ for $\mathrm{OB}$ at baseline $(p=0.070)$. 
Fig. 3. Comparison of sucrose preference. The sucrose preferences of the control (NC) and obesity (OB) groups were measured before and after weight loss by the OB group. Data are shown as a box and whisker plot, with the upper and lower end of the boxes indicating the third and first quartiles of the data, respectively. Median values are represented as the line inside the box. Asterisk indicates significant difference.

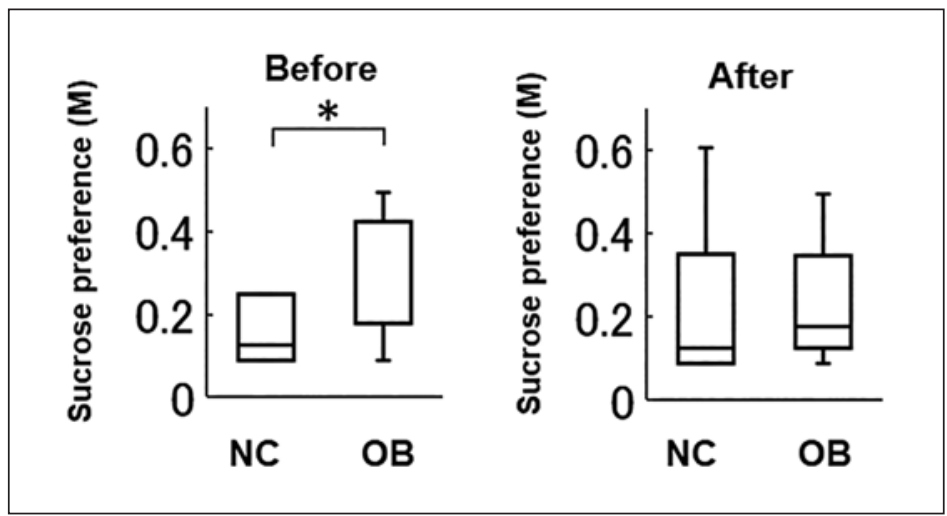

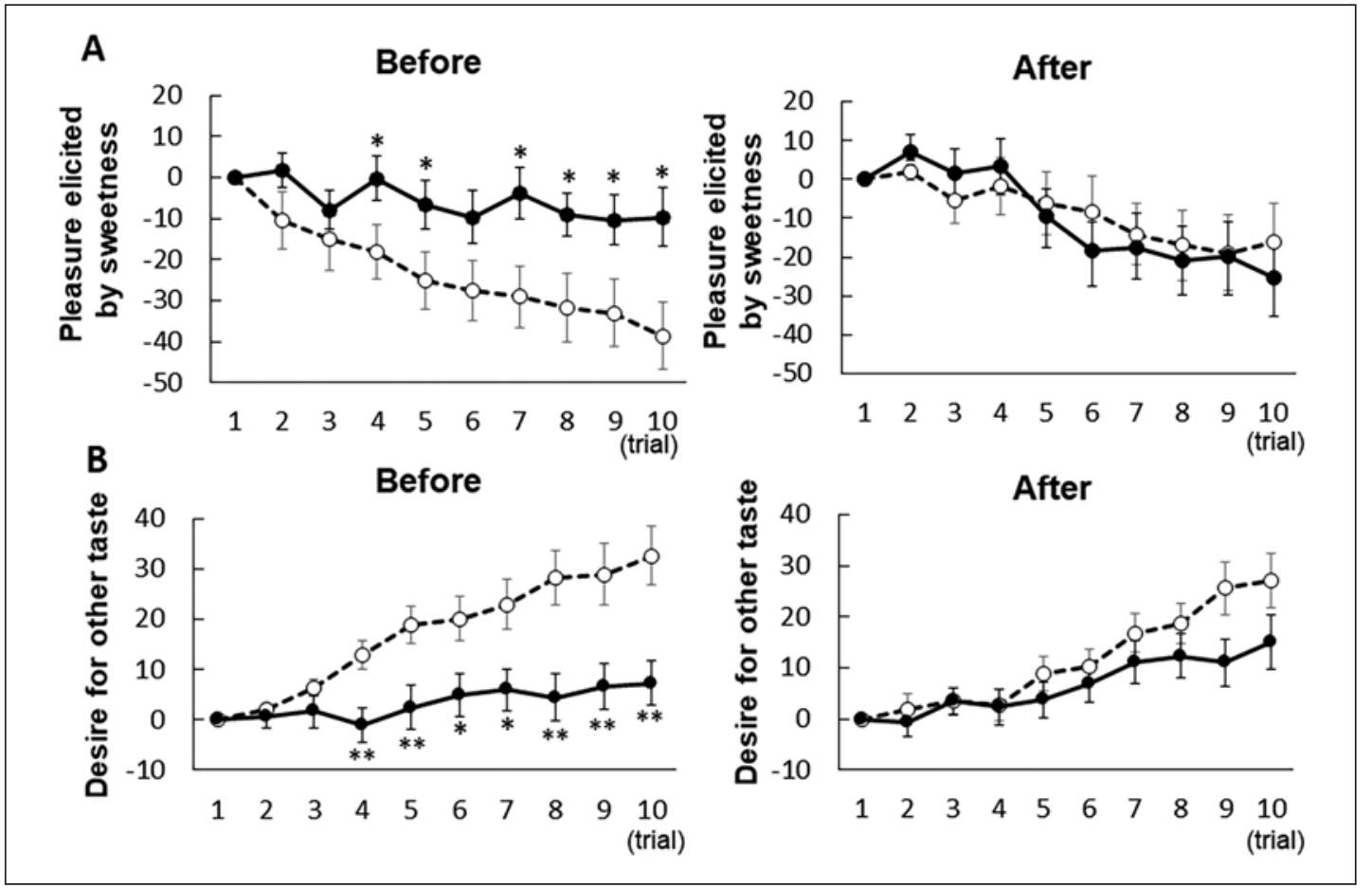

Fig. 4. Kinetics of pleasure elicited by sweetness and the desire for other taste. The control (open circle) and obesity (OB) (closed circle) groups underwent 10 consecutive trials of tasting $24 \% \mathrm{w} / \mathrm{v}$ sucrose solution before and after weight loss by the OB group. The data corresponding to pleasure elicited by sweetness $(\mathbf{A})$ and the desire for other tastes (B) are normalized to their baseline values at the first trial. Data are expressed as mean \pm standard error. Asterisk indicates a simple main effect of significant difference of between-group comparison $\left(* p<0.05,{ }^{* *} p<0.01\right)$.

After weight loss, they were $13.2 \pm 4.1$ for $\mathrm{NC}$ and $19.2 \pm 3.3$ for $\mathrm{OB}(p=0.18)$ (Table 3 shows the mean values of the sweet taste palatability test of both groups). Figure 4A shows the kinetics of the normalized values of "pleasure elicited by sweetness" of both groups. Before weight loss, the ANOVA revealed a significant interaction $(F(4.75,223.31)=2.73, p=0.022)$, and simple main effects of group were detected in all trials after the 4th (Fig. 4A), which suggests that the pleasure was persistent to repeated exposures to sweetness in the OB group. 
Table 3. Mean values of the sweet taste palatability at each trial

\begin{tabular}{|c|c|c|c|c|c|c|c|c|c|c|c|}
\hline & & 1 & 2 & 3 & 4 & 5 & 6 & 7 & 8 & 9 & 10 \\
\hline $\begin{array}{l}\text { Sweetness } \\
\text { pleasure before }\end{array}$ & $\begin{array}{l}\mathrm{NC} \\
\mathrm{OB}\end{array}$ & $\begin{array}{l}11.3 \pm 5.0 \\
-4.8 \pm 6.4\end{array}$ & $\begin{array}{r}0.9 \pm 5.7 \\
-3.1 \pm 6.8\end{array}$ & $\begin{array}{r}-3.7 \pm 6.5 \\
-12.7 \pm 6.0\end{array}$ & $\begin{array}{l}-6.9 \pm 5.8 \\
-5.0 \pm 6.3\end{array}$ & $\begin{array}{l}-13.8 \pm 5.8 \\
-11.3 \pm 6.6\end{array}$ & $\begin{array}{l}-16.2 \pm 5.9 \\
-14.4 \pm 7.4\end{array}$ & $\begin{array}{r}-17.5 \pm 5.9 \\
-8.7 \pm 7.4\end{array}$ & $\begin{array}{l}-20.4 \pm 6.4 \\
-13.8 \pm 6.5\end{array}$ & $\begin{array}{l}-21.6 \pm 6.8 \\
-15.1 \pm 6.8\end{array}$ & $\begin{array}{l}-27.2 \pm 6.7 \\
-14.4 \pm 8.2\end{array}$ \\
\hline $\begin{array}{l}\text { Sweetness } \\
\text { pleasure } \text { after }\end{array}$ & $\begin{array}{l}\mathrm{NC} \\
\mathrm{OB}\end{array}$ & $\begin{array}{l}4.4 \pm 8.1 \\
2.5 \pm 7.6\end{array}$ & $\begin{array}{l}6.3 \pm 7.6 \\
9.6 \pm 5.9\end{array}$ & $\begin{array}{r}-0.9 \pm 8.3 \\
4.3 \pm 6.7\end{array}$ & $\begin{array}{l}2.6 \pm 8.0 \\
5.9 \pm 6.2\end{array}$ & $\begin{array}{l}-1.7 \pm 8.5 \\
-7.0 \pm 5.7\end{array}$ & $\begin{array}{r}-3.8 \pm 9.1 \\
-15.8 \pm 6.1\end{array}$ & $\begin{array}{r}-8.9 \pm 7.8 \\
-15.2 \pm 7.0\end{array}$ & $\begin{array}{l}-12.4 \pm 8.3 \\
-18.3 \pm 7.6\end{array}$ & $\begin{array}{l}-14.5 \pm 9.1 \\
-17.4 \pm 8.0\end{array}$ & $\begin{array}{l}-11.8 \pm 9.3 \\
-23.0 \pm 8.1\end{array}$ \\
\hline $\begin{array}{l}\text { Desire for other } \\
\text { taste before }\end{array}$ & $\begin{array}{l}\mathrm{NC} \\
\mathrm{OB}\end{array}$ & $\begin{array}{r}4.8 \pm 1.4 \\
17.2 \pm 3.7\end{array}$ & $\begin{array}{r}7.0 \pm 1.7 \\
17.9 \pm 3.4\end{array}$ & $\begin{array}{l}11.5 \pm 2.7 \\
18.8 \pm 3.7\end{array}$ & $\begin{array}{l}18.3 \pm 3.4 \\
16.1 \pm 2.9\end{array}$ & $\begin{array}{l}24.7 \pm 4.4 \\
19.6 \pm 3.9\end{array}$ & $\begin{array}{l}26.0 \pm 5.2 \\
22.1 \pm 4.2\end{array}$ & $\begin{array}{l}29.0 \pm 5.4 \\
23.2 \pm 4.3\end{array}$ & $\begin{array}{l}34.6 \pm 5.9 \\
21.6 \pm 4.4\end{array}$ & $\begin{array}{l}35.3 \pm 6.4 \\
23.7 \pm 4.5\end{array}$ & $\begin{array}{l}39.1 \pm 5.8 \\
24.5 \pm 4.5\end{array}$ \\
\hline $\begin{array}{l}\text { Desire for other } \\
\text { taste after }\end{array}$ & $\begin{array}{l}\mathrm{NC} \\
\mathrm{OB}\end{array}$ & $\begin{array}{l}13.2 \pm 4.1 \\
19.2 \pm 3.3\end{array}$ & $\begin{array}{l}15.4 \pm 3.3 \\
18.7 \pm 3.6\end{array}$ & $\begin{array}{l}17.1 \pm 3.4 \\
22.8 \pm 3.9\end{array}$ & $\begin{array}{l}16.2 \pm 3.1 \\
21.7 \pm 4.1\end{array}$ & $\begin{array}{l}22.6 \pm 3.9 \\
23.1 \pm 4.2\end{array}$ & $\begin{array}{l}24.1 \pm 3.8 \\
26.2 \pm 4.6\end{array}$ & $\begin{array}{l}30.9 \pm 3.9 \\
30.5 \pm 5.1\end{array}$ & $\begin{array}{l}33.0 \pm 3.8 \\
31.6 \pm 5.2\end{array}$ & $\begin{array}{l}40.3 \pm 4.3 \\
30.3 \pm 5.2\end{array}$ & $\begin{array}{l}41.8 \pm 4.9 \\
34.4 \pm 5.9\end{array}$ \\
\hline
\end{tabular}

Values are presented as mean \pm SEM.

After weight loss, the interaction was no longer significant $(F(2.47,101.23)=0.87, p=0.44)$, and no significant main effect was detected $(F(1,41)=0.024, p=0.88)$. Figure $4 \mathrm{~B}$ shows the kinetics of the normalized values of "desire for other taste." In the same manner, before intervention the ANOVA revealed significant interaction $(F(2.61,114.83)=7.62, p<0.001)$ and significant simple main effects of group at trials after the 4th (Fig. 4B), which implies that the OB group presented a lower increase in desire for other tastes over time. After the intervention, interaction $(F(2.96,115.26)=2.17, p=0.097)$ and main effect $(F(1,39)=1.44, p=$ $0.24)$ were not significant.

We added a before-after comparison of the sweet taste palatability values of the NC group because they appeared to be changed at follow-up (Fig. 4). Repeated measures ANOVA was applied to compare the slopes, which resulted in no significant interaction (for "pleasure elicited by sweetness" values, $F(3.05,122.06)=1.12, p=0.35$; for "desire for other taste" values, $F(2.33,88.35)=1.18, p=0.32)$.

Association between Sweet Taste Preference and Appetite Hormones

The serum leptin level was significantly higher in the OB group than in the NC group before weight loss $(p<0.001$; Table 1$)$. In contrast, the serum ghrelin levels were not significantly different before the intervention ( $p=0.70$; Table 1$)$. Weight loss significantly decreased the serum leptin level of the OB group $(14.8 \pm 1.6 \mathrm{ng} / \mathrm{mL}, p<0.001)$ to a level comparable to that observed in the NC group.

Subsequently performed Pearson correlation analysis revealed a trend toward a positive correlation between log sucrose concentration and serum leptin level in the OB group before weight loss ( $r=0.378, p=0.052)$. After adjusting for the effects of confounding factors such as BMI, depressive symptom scores, and trait anxiety scores, this trend emerged as a significant association $(r=0.487, p=0.016)$.

\section{Discussion/Conclusion}

In the present study, we showed that our nonsurgical CBT-based weight loss intervention normalized the hedonic component of sweet taste perception of women with obesity to a level comparable to that of the NC group. This suggests that our behavior-focused weight loss caused normalization of central reward arising from sweet taste input. 
Current evidence suggests that taste perception is changed following weight loss induced by both surgical and nonsurgical procedures. Umabiki et al. [31] reported decreased sweet taste threshold after 12-week dietary weight loss by women with obesity, along with an association between changes in the serum leptin level and the sweet threshold. In the recent literature, Burgess et al. [32] reported that liking for sweet and fatty taste decreased after 6 months of dietary weight loss, without change of perceived intensity of taste. Martin et al. [33] studied food preference and revealed that weight loss by low-carbohydrate or low-fat dieting for 2 years reduced the preference and craving for restricted foods. With regard to surgical weight loss, Pepino et al. [6] reported that RYGB and laparoscopic gastric banding (LAGB) decreased perceived sweetness of sucrose and preferred sucrose concentration. RYGB also decreased the pleasure arising from repetitive tasting of the sweetness of sucrose, but LAGB did not change. More recently, they reported that weight loss induced by RYGB and sleeve gastrectomy lowered both preferred sucrose concentration and sweet taste palatability during a sequential sucrose-tasting task, without change of the sensory discriminative component of taste perception [7]. These findings are further supported by a brain imaging study, which showed that RYGB surgery-induced weight loss caused reduced neural activation in the reward circuitry in response to palatable food cues and lowered the hedonic drive to consume palatable foods $[34,35]$.

Interestingly, we found that weight loss induced by the CBT-based approach altered the hedonic but not the sensory discriminative component of sweet taste, which is consistent with findings from the above studies. Together with this current evidence on different types of weight loss procedures, it would seem that the hedonic component is more likely to change than the sensory discriminative component, regardless of the procedure.

Our results indicate the possibility that changes of sweet taste preference and palatability are likely caused by secondary effects of improvement in obesity status. There are several potential mechanisms underlying obesity-associated alteration of taste perception. For example, appetite-regulating hormones, such as leptin, have been proposed as candidate molecules for mediating the beneficial effects of weight loss on taste perception $[3,31]$. In fact, leptin is an adipose tissue hormone that can act in the brain, especially the mesolimbic dopamine system, and subsequently modulate the food reward system [36-38]. The current results of an increased basal serum leptin level in the patients and their association with sucrose preference concentrations imply that leptin activity may be the underlying mechanism of enhanced preference for people with obesity.

Another potential underlying mechanism that should be considered is a decreased amount of sweet taste experience, which can reduce hedonic responses arising from sweet taste input, as previously described [33]. Our participants with obesity significantly decreased the amount of daily sweets consumption, similar to the postsurgical dietary change seen in surgical weight loss participants.

This study is important in that it shows that nonsurgical weight loss, as with the previously well-researched surgical procedures, has a beneficial effect on sweet taste preference and palatability. However, because we did not study subjects who had undergone surgical weight loss, we cannot determine conclusively if the alterations of sweet taste preference and palatability of our participants are the same as those detected in the surgical weight loss studies. In addition, the finding of previous research that sweet taste palatability did not change after LAGB is contrary to our result [6]. It is possible that anatomical change of the gastrointestinal system and subsequent change in satiety-related gut hormones are involved in hedonic perceptional change following surgical weight loss. Additional studies are needed to elucidate the specific mechanisms underlying these shifts following weight loss therapy, as well as its impact on weight maintenance. 
An important limitation of our study must be noted. Taste assessment instruments, such as gLMS, have been widely used as a reliable method for evaluating taste sensation, but include bias because of the subjectivity of the experience of sensation. Furthermore, our palatability values may have been influenced by individual variation. Additionally, it is worth specific mention that sweet taste preference is affected by race and ethnicity [39]. Our Japanese female participants could have presented with different preferences than those of ethnic groups previously reported.

In conclusion, weight loss induced by CBT-based nonsurgical intervention normalized the preference for high sucrose concentration and sweet taste palatability of women with obesity. Further studies are needed to understand the precise mechanisms of this shift.

\section{Acknowledgement}

We thank Ms. Junko Sakaguchi, national registered nutritionist, for her nutritional guidance and assessment; the patients and controls for their cooperation; Junji Kishimoto, associate professor of National University Corporation Kyushu University, Center for Clinical and Transitional Research and Kazufumi Yoshihara, MD, PhD, for their assistance with the statistical calculations, and Ms. Ayako Tomihisa for her assistance with material collection.

\section{Statement of Ethics}

Each participant gave written informed consent. The study protocol was approved by the Institutional Review Board of Kyushu University Hospital (No. 765). The study was registered in a public trials registry before first patient enrolment (UMIN ID: 000021868).

\section{Disclosure Statement}

The authors have no conflicts of interest to declare.

\section{Funding Sources}

All phases of this study were supported by a KAKENHI Grant-in-Aid for Scientific Research (B; Grant JP 17K09306 to T. Nozaki. and Grant JP 16 H05278 to N.S.), a KAKENHI Grant-in-Aid for Exploratory Research (Grant JP 16K15413 to N.S.), and a KAKENHI Grant-in-Aid for Scientific Research on Innovative Areas ("Willdynamics" Grant 16H06404 to N.S.) from the Japan Society for the Promotion of Science (JSPS).

\section{Author Contributions}

T. Nishihara and R. Sawamoto designed and carried out the experiments. T. Nozaki and R. Sawamoto conducted the weight loss intervention. T. Nishihara and T. Nozaki conceived the experiments and analyzed the data. All authors were involved in writing the paper and gave final approval of the submitted and published versions. 
Nishihara et al.: Effects of Weight Loss on Sweet Taste Preference and Palatability

\section{References}

1 Luger M, Lafontan M, Bes-Rastrollo M, Winzer E, Yumuk V, Farpour-Lambert N. Sugar-Sweetened Beverages and Weight Gain in Children and Adults: A Systematic Review from 2013 to 2015 and a Comparison with Previous Studies. Obes Facts. 2017;10(6):674-93.

2 Jayasinghe SN, Kruger R, Walsh DC, Cao G, Rivers S, Richter M, et al. Is Sweet Taste Perception Associated with Sweet Food Liking and Intake? Nutrients. 2017 Jul;9(7):E750.

3 Berthoud HR, Zheng H. Modulation of taste responsiveness and food preference by obesity and weight loss. Physiol Behav. 2012 Nov;107(4):527-32.

4 Bartoshuk LM, Duffy VB, Green BG, Hoffman HJ, Ko CW, Lucchina LA, et al. Valid across-group comparisons with labeled scales: the gLMS versus magnitude matching. Physiol Behav. 2004 Aug;82(1):109-14.

5 Bartoshuk LM, Duffy VB, Hayes JE, Moskowitz HR, Snyder DJ. Psychophysics of sweet and fat perception in obesity: problems, solutions and new perspectives. Philos Trans R Soc Lond B Biol Sci. 2006 Jul;361(1471): 1137-48.

6 Pepino MY, Bradley D, Eagon JC, Sullivan S, Abumrad NA, Klein S. Changes in taste perception and eating behavior after bariatric surgery-induced weight loss in women. Obesity (Silver Spring). 2014 May;22(5):E1320.

7 Nance K, Eagon JC, Klein S, Pepino MY. Effects of Sleeve Gastrectomy vs. Roux-en-Y Gastric Bypass on Eating Behavior and Sweet Taste Perception in Subjects with Obesity. Nutrients. 2017 Dec;10(1):E18.

8 Kuga M, Ikeda M, Suzuki K. Gustatory changes associated with the menstrual cycle. Physiol Behav. 1999 Apr; 66(2):317-22.

9 Pepino MY, Mennella JA. Effects of cigarette smoking and family history of alcoholism on sweet taste perception and food cravings in women. Alcohol Clin Exp Res. 2007 Nov;31(11):1891-9.

10 Pribitkin E, Rosenthal MD, Cowart BJ. Prevalence and causes of severe taste loss in a chemosensory clinic population. Ann Otol Rhinol Laryngol. 2003 Nov;112(11):971-8.

11 Pepino MY, Finkbeiner S, Beauchamp GK, Mennella JA. Obese women have lower monosodium glutamate taste sensitivity and prefer higher concentrations than do normal-weight women. Obesity (Silver Spring). 2010 May;18(5):959-65.

12 Coldwell SE, Mennella JA, Duffy VB, Pelchat ML, Griffith JW, Smutzer G, et al. Gustation assessment using the NIH Toolbox. Neurology. 2013 Mar;80(11 Suppl 3):S20-4.

13 Pepino MY, Mennella JA. Habituation to the pleasure elicited by sweetness in lean and obese women. Appetite. 2012 Jun;58(3):800-5.

14 Lanier SA, Hayes JE, Duffy VB. Sweet and bitter tastes of alcoholic beverages mediate alcohol intake in of-age undergraduates. Physiol Behav. 2005 Jan;83(5):821-31.

15 Nagai M, Matsumoto S, Endo J, Sakamoto R, Wada M. Sweet taste threshold for sucrose inversely correlates with depression symptoms in female college students in the luteal phase. Physiol Behav. 2015 Mar;141:92-6.

16 Shima S, Shikano T, Kitamura T, Asai M. A new self-report depression scale [in Japanese]. Clin Psychiatry. 1985;27:717-23.

17 Radloff L. The CES-D Scale: A Self-Report Depression Scale for Research in the General Population. Appl Psychol Meas. 1977;1(3):385-401.

18 Nakazato K, Mizuguchi K. Development and validation of Japanese version of State-Trait Anxiety Inventory: A study with female subjects [in Japanese]. Jpn J Psychosom Med. 1982;22(2):107-12.

19 Spielberger C, Gorsuch R, Lushene R, Vagg P, Jacobs G. Manual for the state trait anxiety inventory (STAI). Palo Alto: Consulting Psychologists Press; 1983.

20 Gormally J, Black S, Daston S, Rardin D. The assessment of binge eating severity among obese persons. Addict Behav. 1982;7(1):47-55.

21 Adachi T, Fujii K, Yamagami T. Responses regarding restrained eating on the Three-Factor Eating Questionnaire and weight loss [in Japanese]. Jpn J Behav Ther. 1992 Sep;18(2):140-8.

22 Stunkard AJ, Messick S. The three-factor eating questionnaire to measure dietary restraint, disinhibition and hunger. J Psychosom Res. 1985;29(1):71-83.

23 Imada S. Psychological studies about eating behavior (3): a Japanese version of the Dutch Eating Behavior Questionnaire (DEBQ). Stud Humanit Sci. 1994 Mar;34(2):281-91.

24 van Strien T, Frijters ER, Bergers GP, Defares PB. The Dutch Eating Behavior Questionnaire (DEBQ) for assessment of restrained, emotional, and external eating behavior. Int J Eat Disord. 1986 Feb;5(2):295-315.

25 Cooper Z, Fairburn CG. A new cognitive behavioural approach to the treatment of obesity. Behav Res Ther. 2001 May;39(5):499-511.

26 Nozaki T, Sawamoto R, Sudo N. Cognitive behavioral therapy for obesity [in Japanese]. Nihon Rinsho. 2013 Feb;71(2):329-34.

27 Kitamura S. Diet therapy and food exchange lists for diabetic patients. Diabetes Res Clin Pract. 1994 Oct;24 Suppl:S233-40.

28 Japan Society for the Study of Obesity. Guideline for the management of obesity disease 2016. Tokyo: Life Science Publication; 2016. [in Japanese].

29 Ministry of Health, Labour and Welfare. Nutrition and dietary habits, Health Japan 21 [in Japanese]. [accessed 2019 June 9]. Available from: https://www.mhlw.go.jp/www1/topics/kenko21_11/b1.html. 
Sawamoto R, Nozaki T, Nishihara T, Furukawa T, Hata T, Komaki G, et al. Predictors of successful long-term weight loss maintenance: a two-year follow-up. Biopsychosoc Med. 2017 Jun;11(1):14.

31 Umabiki M, Tsuzaki K, Kotani K, Nagai N, Sano Y, Matsuoka Y, et al. The improvement of sweet taste sensitivity with decrease in serum leptin levels during weight loss in obese females. Tohoku J Exp Med. 2010 Apr;220(4): 267-71.

32 Burgess B, Rao SP, Tepper BJ. Changes in liking for sweet and fatty foods following weight loss in women are related to prop phenotype but not to diet. Obesity (Silver Spring). 2016 Sep;24(9):1867-73.

33 Martin CK, Rosenbaum D, Han H, Geiselman PJ, Wyatt HR, Hill JO, et al. Change in food cravings, food preferences, and appetite during a low-carbohydrate and low-fat diet. Obesity (Silver Spring). 2011 Oct;19(10): 1963-70.

34 Ochner CN, Kwok Y, Conceição E, Pantazatos SP, Puma LM, Carnell S, et al. Selective reduction in neural responses to high calorie foods following gastric bypass surgery. Ann Surg. 2011 Mar;253(3):502-7.

35 Scholtz S, Miras AD, Chhina N, Prechtl CG, Sleeth ML, Daud NM, et al. Obese patients after gastric bypass surgery have lower brain-hedonic responses to food than after gastric banding. Gut. 2014 Jun;63(6):891-902.

36 Hommel JD, Trinko R, Sears RM, Georgescu D, Liu ZW, Gao XB, et al. Leptin receptor signaling in midbrain dopamine neurons regulates feeding. Neuron. 2006 Sep;51(6):801-10.

37 Fulton S, Pissios P, Manchon RP, Stiles L, Frank L, Pothos EN, et al. Leptin regulation of the mesoaccumbens dopamine pathway. Neuron. 2006 Sep;51(6):811-22.

38 Domingos AI, Vaynshteyn J, Voss HU, Ren X, Gradinaru V, Zang F, et al. Leptin regulates the reward value of nutrient. Nat Neurosci. 2011 Nov;14(12):1562-8.

39 Pepino MY, Mennella JA. Factors contributing to individual differences in sucrose preference. Chem Senses. 2005 Jan;30 Suppl 1:i319-20. 